\title{
Spéciation et dosimétrie interne : des espèces chimiques aux modèles dosimétriques
}

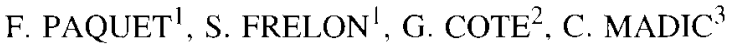

RÉSUMÉ Les études de spéciation correspondent à la détermination des espèces chimiques d'un élément et à l'analyse de leur distribution dans un échantillon ou un milieu donné. Ces êtudes sont nécessaires pour mieux décrire, comprendre et prédire les biocinétiques des éléments traces. Dans le cas d'une contamination interne par des radionucléides, les études de spéciation permettent de mieux comprendre certains mécanismes d'absorption et de transfert. Elles contribuent de fait à améliorer les modèles biocinétiques et dosimétriques des radionucléides. Différentes approches peuvent être utilisées pour l'étude de la spéciation des radionucléides, en fonction du niveau d'étude demandé et du degré d'incertitude toléré. Parmi celles-ci, on distingue une approche théorique par modélisation et une approche expérimentale qui sont toutes deux complémentaires. Cet article résume et décrit ce qui est actuellement connu sur la spéciation des actinides dans le sang, le tractus gastrointestinal (TGI), le foie et les os mais aussi sur les conséquences induites en radiotoxicologie. Dans le futur, les recherches devront être focalisées sur certains tissus spécifiques afin de répondre aux principales questions de la dosimétrie interne.

ABSTRACT Speciation and Internal dosimetry: from chemical species to dosimetric models.

Speciation studies refer to the distribution of species in a particular sample or matrix. These studies are necessary to improve the description, understanding and prediction of trace element kinetics and toxicity. In case of internal contamination with radionuclides, speciation studies could help to improve both the biokinetic and dosimetric models for radionuclides. There are different methods to approach the speciation of radionuclide in a biological system, depending on the degree of accuracy needed and the level of uncertainties accepted. Among them, computer modelling and experimental determination are complementary approaches. This paper describes what is known about speciation of actinides in blood, GI-tract, liver and skeleton and of their consequences in terms of internal dosimetry. The conclusion is that such studies provide very valuable data and should be targeted in the future on some specific tissues and biomolecules.

\footnotetext{
IRSN. Luboratoire de radiotoxicologie expérimentale. B.P. I66, 26702 Pierrelatte Cedex, France.

École nationale supérieure de chimie de Paris (UMR 7575 du CNRS), 11 rue Pierre et Murie Curie, 75005 Paris, France.

3 CEA Saclay, DEN, 91/91 Gif-sur-Yvette, France.
} 


\section{Introduction}

Les études de spéciation ${ }^{4}$ correspondent à la détermination des espèces chimiques d'un élément et à l'analyse de leur distribution dans un échantillon ou un milieu donné. Ce concept a été intégré en biologie dans les années 50 au travers des recherches menées en pharmacologie et en écotoxicologie dont l'un des objectifs était de définir la toxicité des éléments. Cette toxicité était décrite à l'époque comme étant fortement dépendante de la forme chimique d'un élément considéré et largement accrue dans le cas d'un ion métallique libre en solution par rapport à sa forme complexée (voir synthèse dans Simkiss et Taylor, 2001). De nos jours, il est admis que ces relations sont plus complexes mais que, de façon générale, la spéciation des éléments influence leur stabilité et mobilité dans les sols, ainsi que leurs bio-disponibilité, carcinogénécité, mutagénécité, métabolisme et bio-cinétique dans les organismes vivants.

Il a ainsi été démontré que la filtration d'un ion métallique par les reins et donc son excrétion et sa distribution dans le corps, est très différente si celui-ci est lié à une protéine ou s'il est libre dans le milieu (Savory et al., 1983). En toxicologie, il a été montré que les formes ioniques libres des éléments étaient de façon générale plus toxiques que les formes complexées mais aussi que certaines formes complexées étaient plus toxiques que d'autres. Des expériences réalisées sur l'arsenic montrent ainsi que les formes organiques telles que l'arsénobétaine $\left(\mathrm{As}{ }^{(\mathrm{V})} \mathrm{C}_{5} \mathrm{O}_{2} \mathrm{H}_{11}\right)$, rencontrées dans la nourriture marine, sont inoffensives tandis que les formes inorganiques $\mathrm{AsO}_{4}{ }^{3-}$ et $\mathrm{AsO}_{3}$ sont très toxiques (Sabbioni et al., 1991). De même, la complexation d'un élément par des métallothionéines, qui sont des protéines induites en réponse à un stress, a pour conséquence de prévenir la toxicité de plusieurs métaux dont le zinc, le cadmium, le cuivre et le mercure alors que ceux-ci restent toxiques lorsqu'ils sont sous d'autres formes (Goyer, 1983).

En marge de ces phénomènes de complexation, il a également été montré que la présence d'autres métaux peut fortement influencer les biocinétiques et la toxicité d'un élément. Plusieurs exemples montrent l'influence positive d'une déficience en fer sur l'absorption du cadmium (Flanagan et al., 1978), le rôle protecteur de l'arsenic contre la toxicité de nombreuses espèces de sélénium dans plusieurs composés (Jonnalagadda et Rao, 1993) et aussi l'effet protecteur du sélénium contre la toxicité de nombreux métaux lourds (Whanger, 1992).

\footnotetext{
4 Le terme spéciation a été utilisé dans le passé pour décrire différentes notions, incluant la distribution evou les transformations des espèces chimiques mais aussi les activités analytiques visant à déterminer la présence et la concentration de ces espèces. Dans ce document, le terme spéciation est utilisé pour décrire la distribution des espèces dans un échantillon ou une matrice donnée tandis que les études de spéciation font référence à l'identification puis à la quantification de la forme physico-chimique d'un élément (état Rédox, structure du complexe, stochiométrie, nature organique ou inorganique des ligands).
} 
Toutes ces caractéristiques ont été étudiées en sciences médicales et environnementales telles que la pharmacologie, la médecine, l'agronomie, l'écotoxicologie et la géochimie et ont permis de fournir des données à la fois aux scientifiques pour leur permettre de comprendre les phénomènes biologiques et au législateur pour définir des limites d'exposition. En radioprotection, ce concept de spéciation n'est que peu utilisé. Ce manque d'intérêt est préjudiciable car la spéciation donne des informations essentielles sur la biochimie et la chimie des éléments, indispensables pour établir les modèles biocinétiques et dosimétriques utilisés en radioprotection et pour améliorer les procédures utilisées dans le traitement des contaminations internes. La revue présentée ici fait le point sur les principales avancées dans ce domaine et donne quelques perspectives au niveau des recherches qu'il conviendrait de mener.

\section{Méthodes d'analyse de la spéciation d'un élément}

Avant d'aborder les résultats obtenus sur la spéciation des radionucléides dans les tissus biologiques, il semble indispensable de faire un point sur les approches méthodologiques couramment utilisées. Dans ce domaine, la représentativité et la validité des données obtenues dépendront largement de la méthode employée. II est donc nécessaire de définir avant toute étude, à la fois la nature des données recherchées (composition isotopique, état d'oxydation, nature des complexes, etc.) et le niveau d'incertitudes acceptable (Templeton et al., 2000). De plus, il est nécessaire de savoir si les objectifs de l'étude visent à obtenir une analyse de la distribution des espèces à l'équilibre ou si une notion dynamique, intégrant les processus de transport et de transformation doit être introduite.

Ces dernières décennies, deux approches complémentaires ont été utilisées. L'une d'elle consiste à étudier la spéciation d'un élément par modélisation informatique et l'autre à déterminer la nature des diverses espèces présentes par des techniques analytiques.

\subsection{Approche de la spéciation par modélisation}

La modélisation informatique a pour objectif de calculer la distribution des espèces dans un milieu donné pour un élément donné. Ces calculs intègrent généralement les constantes thermodynamiques de solubilité, de précipitation, de formation de complexes, des réactions acido-basiques et des réactions d'oxydo-réduction. Ils reposent sur un calcul du coefficient de complexation de l'ion métallique étudié avec tous les ligands présents et en fonction du $\mathrm{pH}$. Peu à peu, des codes de calculs spécifiques ont été produits afin de se soustraire à cette approche un peu laborieuse. Malheureusement, il n'existe à ce jour aucun code capable d'estimer 
les interactions entre les éléments et l'ensemble des ligands présents dans les systèmes biologiques. Des codes initialement conçus pour des applications géologiques et environnementales ont été dérivés pour des utilisations en milieu biologique (MINEQL ou FITEQL (Westall et al., 1986), PHREEQE (Parkhurst et al., 1980), REACT (Bethke, 1996), JESS (May et Murray, 1991) et CHESS (Van der Lee, 1998), mais de nombreux progrès restent à faire puisque les constantes d'affinité entre les protéines - principaux ligands biologiques - et les métaux ne sont pas inclues dans les bases de données disponibles et que les calculs portent sur un système à l'équilibre. Ces points soulignent le fait que les caractéristiques des milieux biologiques, dans lesquels les réactions biochimiques sont susceptibles d'être initiées, doivent être définies puis intégrées dans les codes de calcul avec la plus grande précision possible.

Par ailleurs, les bases thermodynamiques desquelles sont extraites les données utilisées pour les calculs doivent être les plus fiables possible. Ces données varient souvent d'une base à une autre et, le plus souvent, ont été déterminées pour une température et une force ionique constantes (la plupart du temps $\mathrm{T}=25^{\circ} \mathrm{C}$ et $\mathrm{I}=0 \mathrm{M})$, différentes de celles des mammifères $\left(\mathrm{T}=37^{\circ} \mathrm{C}\right.$ et $\left.\mathrm{I}=0,15 \mathrm{M}\right)$. De plus, certaines données ont été estimées à partir d'analogies chimiques entre deux éléments. Les incertitudes, liées à ces analogies doivent être prises en compte. Certaines bases de données, comme celle publiée par l'Agence internationale à l'énergie atomique (Fuger et al., 1992), celle de l'Agence de l'énergie nucléaire (OECD/NEA) (Grenthe et al., 1992) ou encore la base IUPAC des constantes de stabilité (SC-database ; Powel, 1999) présentent une revue critique des données publiées et indiquent les incertitudes à intégrer dans les calculs.

En conclusion, la spéciation par modélisation informatique ne doit pas être utilisée comme seul outil pour déterminer la spéciation dans les milieux biologiques mais seulement afin d'obtenir une première estimation de la distribution des espèces à l'équilibre. De plus, présence d'espèces n'est donnée que pour des systèmes à l'équilibre et ne prennent pas en compte les cinétiques des réactions. Ces données doivent alors être validées par des données expérimentales obtenues par analyse des complexes métalliques. Les méthodes utilisées doivent cibler, avec un minimum de perturbation du système et de son profil de spéciation, l'extraction, la séparation, l'identification et la quantification des produits de réaction des métaux et des éléments non métalliques avec les composés des tissus et des cellules.

\subsection{Approche expérimentale de la spéciation}

L'approche expérimentale est basée sur l'utilisation couplée d'une technique séparative et d'une détection sensible et spécifique. Deux approches doivent être distinguées selon que les espèces et complexes à détecter et mesurer soient déjà identifiés ou non. 
Dans le premier cas (complexes connus) des solutions étalon des espèces et complexes à doser sont préparées et utilisées afin de calibrer les systèmes de détection (Guilmette et Edison, 1992 ; Van den Heuvel et al., 1995). Une séparation est alors effectuée, le plus souvent soit en chromatographie (phase inverse, exclusion de taille, échange de cation ou d'anion (Lehmann et al., 1983 ; Paquet et al., 1998b ; Schuppler et al., 1988), soit en électrophorèse bidimensionnelle ou capillaire (Scapolan et al., 1997 ; Taylor et al., 1987 ; Turner et Taylor, 1968). Les éléments sont ensuite détectés par diverses méthodes incluant, pour les métaux stables, la spectrométrie d'absorption atomique avec ionisation électrothermique (ET-AAS) ou ionisation induite par une torche à plasma (ICP-AES), ou encore la spectrométrie de masse avec ionisation induite par une torche à plasma (ICP-MS) (Martell et Calvin, 1959). Pour les radioéléments, la détection, très fine, peut se faire par comptage des différentes émissions radioactives $(\alpha, \beta, \gamma)$. Ces dernières années, de nombreuses évolutions ont eu lieu au niveau des outils de détection. En particulier, le remplacement du mode d'ionisation ET (électrothermique) par l'ICP (induite par une torche à plasma) a permis d'abaisser les limites de détection des éléments mais aussi leur temps d'analyse car ces appareils sont plus sensibles et souvent directement couplés à la méthode de séparation.

Dans le second cas, relatif à des espèces ou des complexes non-identifiés, une caractérisation moléculaire ou élémentaire des composés est nécessaire. Les méthodes de détection seront alors différentes et plus spécifiques car elles nécessitent de détecter non seulement l'élément mais aussi toute la structure dans laquelle il se trouve (Eisele et Erickson, 1985 ; McInroy et al., 1985 ; Sasser et al., 1986 ; Taylor et al., 1991 ; Zalikin et Nisimov, 1990). Des méthodes très résolutives et très sensibles telles que la spectrométrie de masse avec une ionisation par électrospray (ESI-MS), la fluorescence induite par laser et résolue en temps (TRLIF) ou encore les spectroscopies d'absorption X (EXAFS et XANES) seront alors utilisées pour la caractérisation (à différents niveaux suivant la technique utilisée et suivant les composés étudiés).

L'utilisation de ces techniques implique de postuler qu'un métal et une protéine apparaissant ensemble dans une fraction analysée sont associés dans l'échantillon d'origine. Ceci n'est malheureusement pas toujours vrai et il est important de vérifier qu'aucune dissociation et/ou recombinaison entre l'élément étudié et le ligand biologique n'est intervenue. Ces phénomènes inopportuns peuvent être dus aux conditions expérimentales et sont extrêmement fréquents (Toohey et Essling, 1980). Des changements au niveau de l'équilibre des espèces peuvent être dus à un changement de $\mathrm{pH}$ imposé par la procédure analytique mais aussi aux propriétés intrinsèques des milieux utilisés. De plus, quand un échantillon complexe, tel que le plasma sanguin ou le foie est fractionné, chacune des fractions obtenues peut contenir de nombreux ligands potentiels et 
l'association univoque d'un radionucléide avec un composé donné ne peut pas être démontrée (Cornelis et al., 1998).

En conclusion, l'essor et le perfectionnement des outils d'analyse permettra une détection des traces (éléments eux-mêmes ou complexes) de plus en plus précise, sensible et fiable. L'étape limitante de l'étude de spéciation reste alors l'extraction du composé d'une matrice biologique, en conservant intact le profil de distribution des espèces.

\section{Bilan des connaissances sur la spéciation des actinides chez les animaux}

Les recherches réalisées sur la spéciation des actinides peuvent être arbitrairement regroupées en quatre thématiques visant à améliorer respectivement les connaissances en biocinétique, toxicologie, dosimétrie et décorporation des éléments.

\subsection{Amélioration des modèles biocinétiques}

La plupart des études de biocinétique ont été focalisées sur le sang eu égard au fait que les biocinétiques générales des radionucléides sont grandement influencées par leur comportement dans ce tissu. Une revue publiée par Taylor a montré que, dans le sang, la plupart des actinides sont principalement liés à la transferrine (Tf), protéine de transport du fer. La liaison est apparemment plus forte dans le cas de $\mathrm{Th}(\mathrm{IV}), \mathrm{Pu}(\mathrm{IV}), \mathrm{Np}(\mathrm{V})$ et $\mathrm{Pa}(\mathrm{V})$ par rapport aux actinides trivalents et à l'uranium hexavalent (Taylor, 1989). Ceci a des conséquences importantes étant donné que la constante de stabilité des complexes actinide-Tf apparaît comme étant inversement proportionnelle, à la fois, à la rapidité de distribution des actinides dans les tissus cible et à leur excrétion urinaire (Durbin et al., 1997a).

Des expériences de spéciation similaires ont été réalisées au niveau d'organes de dépôt comme le foie (Boocock et al., 1970 ; Bruenger et al., 1971 ; Seidel et al., 1985), ou les os (Tseveleva, 1960) ou sur des excrétas (Stradling et al., 1976 ; Popplewell et al., 1975). Bien que les ligands spécifiques de chaque tissu restent à définir, un comportement schématique des actinides après leur entrée dans la cellule peut-être dressé. Les premières études réalisées sur le foie de rongeurs ont montré que ${ }^{241} \mathrm{Am}(\mathrm{III})$ et ${ }^{249} \mathrm{Cf}(\mathrm{III})$ se déposent préférentiellement dans les lysosomes des cellules (Bruenger et al., 1972 ; Sutterlin et al., 1984) tandis que ${ }^{237} \mathrm{~Np}(\mathrm{~V})$ et ${ }^{239} \mathrm{Pu}(\mathrm{IV})$ se déposent à la fois dans le noyau et les lysosomes (Schuler et Taylor, 1987 ; Paquet et al., 1995). Des études complémentaires effectuées avec ${ }^{241} \mathrm{Am}$ (III), ${ }^{239} \mathrm{Pu}$ (IV) et ${ }^{237} \mathrm{~Np}$ (V) sur du foie de singe ont confirmé ces résultats (Paquet et al., 1998b). Pour les trois radionucléides ${ }^{237} \mathrm{~Np}(\mathrm{~V}),{ }^{239} \mathrm{Pu}(\mathrm{IV})$ et ${ }^{24 I} \mathrm{Am}(\mathrm{III})$, le cytosol semble être le site de dépôt privilégié 24 heures après une 
exposition aiguë tandis que le noyau et les lysosomes sont impliqués plus tard (10 jours après exposition). Dans le cytosol, Pu et Am sont principalement liés à la ferritine quel que soit le moment du sacrifice. Par contre, à la fois chez les rats et les singes, $\mathrm{Np}$ serait lié, juste après l'exposition (1-24 h) conjointement à la ferritine et à la calmoduline tandis qu'après une plus longue période (1-40 jours) il serait majoritairement complexé par la ferritine et d'autres composés de masse molaire élevée.

Les recherches sur les os ont été moins développées car les études sur le squelette impliquent une décalcification de la matrice minérale, pouvant induire un changement de la forme chimique de l'élément étudié. Les premiers résultats ont montré que, après injection chez les rats, l'élément plutonium se fixe principalement sur les protéines et que 65-80\% de l'élément est lié au collagène (Tseveleva, 1960). D'autres, au contraire, montrent que les éléments transuraniens se fixent principalement sur la phase minérale de l'os (Masse et al., 1973). Ces contradictions montrent que des recherches complémentaires dans ce domaine doivent être effectuées et ainsi fournir des informations utiles sur les cinétiques de dépôt et de recyclage des radionucléides dans l'os.

L'absorption dans l'intestin dépend largement de la forme chimique du radionucléide dans la nourriture et des propriétés chimiques du suc gastrique dans le tractus gastro-intestinal (conditions rédox, présence de ligands, etc.). Par conséquent, des données de spéciation sur les radionucléides dans la nourriture ou le tractus gastro-intestinal pourraient donner des informations précises sur leur transfert potentiel vers le sang. Des études récentes de modélisation effectuées au sein du laboratoire ont montré que dans la salive, après son entrée sous forme $\mathrm{UO}_{2}{ }^{2+}$, l'uranium existerait à $\mathrm{pH} 7,5$ à la fois sous forme tétravalent et hexavalent (Lana, 2000). U(IV) serait principalement sous forme $\mathrm{UF}_{6}{ }^{2-}$ tandis que U(VI) pourrait être rencontré sous les formes $\mathrm{UO}_{2} \mathrm{PO}_{4}^{-}, \mathrm{UO}_{2}\left(\mathrm{CO}_{3}\right)_{2}{ }^{2-}$ et $\mathrm{UO}_{2}\left(\mathrm{CO}_{3}\right)_{3}{ }^{4-}$. Ces études ont montré qu'après ingestion de $10^{-6} \mathrm{M} \mathrm{d}^{\prime} \mathrm{UO}_{2}{ }^{2+}$ en solution dans l'eau, cinq formes potentielles sont susceptibles d'être transférées vers l'œsophage et l'estomac. Des études similaires réalisées avec de l'uranium dans le duodénum, dans lequel l'absorption est susceptible d'avoir lieu, ont montré qu'à $\mathrm{pH} 7,5$ c'est la forme $\mathrm{UO}_{2}\left(\mathrm{CO}_{3}\right)_{5}{ }^{8-}$ qui est prédominante (Jones et al., 2002).

L'ensemble de ces données permet une meilleure description de l'absorption des actinides en fonction de leurs formes chimiques et devrait donc participer à l'évolution des modèles biocinétiques.

\subsection{Amélioration des modèles toxicologiques}

Du point de vue toxicologique, la détermination de la forme chimique des radionucléides peut apparaître moins importante que dans le cas des métaux classiques 


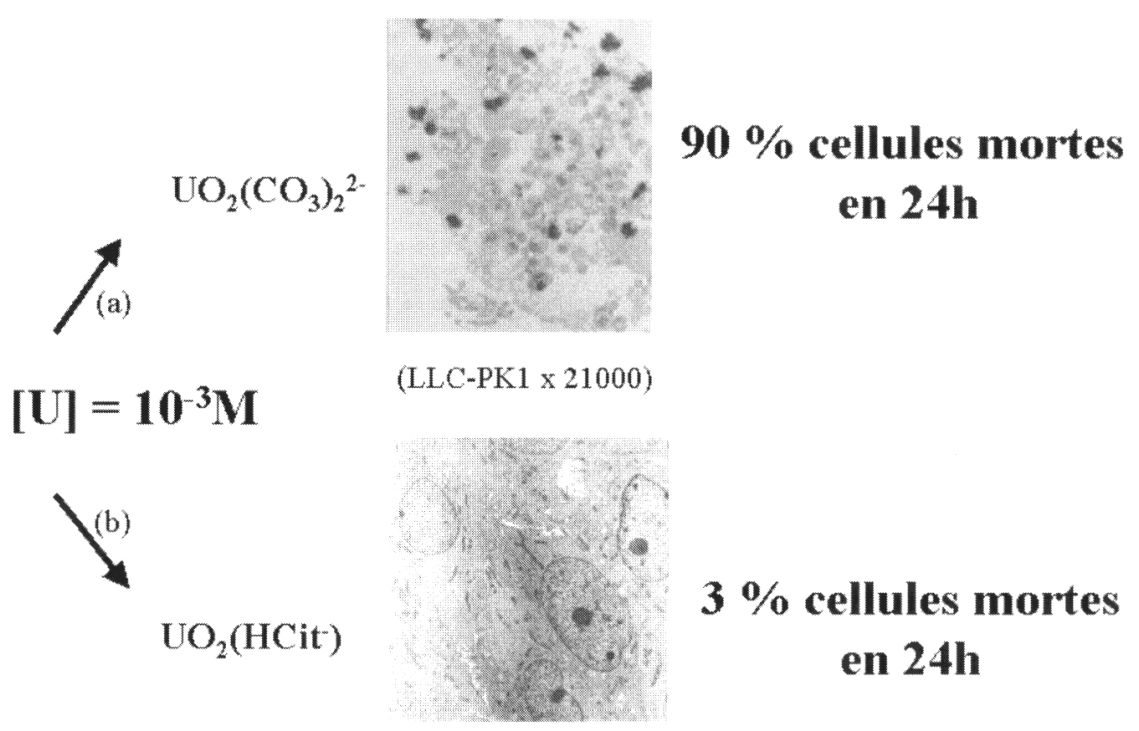

Figure 1 - Influence de la spéciation de l'uranium sur sa toxicité au niveau de cellules rénales (d'après les données de Mirto et al., 1999). Contamination in vitro de cellules LLC-PK1 par une solution d'uranium(VI)-bicarbonate ou d'uranium(VI)-citrate. Bilan de la mortalité et analyse en microscopie à $T+24$ heures.

Influence of uranium speciation on its toxicity in kidneys at a cellular level (from Mirto et al., 1999). In vitro contamination of LLC-PK1 cells by uranium (VI)-bicarbonate or uranium (VI)citrate. Microscopic and mortality analysis at $T+24$ hours.

car l'émission de rayonnement et donc la radiotoxicité des radionucléides est indépendante de leur forme chimique. Selon cette analyse, les études de spéciation ne devraient être entreprises que pour des radionucléides connus comme étant également chimiotoxiques. Cette analyse n'est que partiellement exacte. Des études récentes ont montré que la forme chimique des radionucléides influence leur chimiotoxicité mais aussi leur solubilité, pouvant parfois conduire à des précipités spécifiques à l'intérieur des cellules. Ceci est illustré par le travail de Mirto (Mirto et al., 1999) qui a étudié les interactions entre l'uranium et les cellules rénales (LLC-PK1). Il a montré que le complexe $\mathrm{UO}_{2}\left(\mathrm{CO}_{3}\right)_{2}{ }^{2-}$, une fois pénétré à l'intérieur du compartiment cytoplasmique, précipite sous forme d'aiguilles de phosphate d'uranyle (Fig. 1) et induit une réponse toxique en fonction de la concentration. Au contraire, l'uranium, introduit sous forme citrate ne précipite pas et n'induit aucune toxicité, quelle que soit la concentration utilisée.

La précipitation des radionucléides a pour conséquence leur accumulation très localisée dans les cellules. Ces concentrations d'éléments, à la fois radio- et 
chimio-toxiques, peuvent entraîner de la part d'un groupe de cellules une réponse biologique spécifique capable de causer des anomalies fonctionnelles et structurales et ainsi augmenter la toxicité globale.

En conclusion, la spéciation peut influencer directement la toxicité chimique de certains radionucléides (due à la nature des espèces) et, indirectement, à la fois leur radio- et leur chimio-toxicité (via une accumulation microscopique des radionucléides).

\subsection{Amélioration des modèles dosimétriques}

Les modèles dosimétriques sont basés sur une distribution homogène des radionucléides dans les tissus. L'exemple cité ci-avant montre que la distribution des éléments peut être fortement hétérogène avec une concentration notable dans certaines structures tissulaires (Fig. 2) ou cellulaires. D'autres études, réalisées sur le neptunium ou le plutonium ont montré de façon similaire que ces radionucléides sont concentrés dans certains types de cellules hépatiques, voire dans certaines structures cellulaires telles que le noyau ou les lysosomes (Paquet et al., 1996). Ces accumulations très localisées entraînent un dépôt d'énergie hétérogène et impliquent que la dose reçue par les cellules cibles n'est pas correctement modélisée par la CIPR.

Les études de spéciation peuvent aider à mieux décrire la microlocalisation des radionucléides par rapport aux cellules souche ou aux molécules cible. Elles peuvent ainsi fournir des données devant être intégrées dans les modèles dosimétriques, permettant une meilleure évaluation de la dosimétrie et par conséquent du risque de cancer radio-induit.

\subsection{Amélioration des traitements des contaminations internes}

Les chélateurs artificiels, conçus pour éliminer les radionucléides après incorporation doivent complexer l'élément dans l'organisme de manière compétitive par rapport aux ligands naturels. La connaissance des interactions entre les radionucléides et les ligands biologiques naturels est donc un préalable à la conception de ces molécules. Les études de spéciation peuvent donc apporter des informations sur la structure, l'affinité et la période biologique que doivent avoir ces chélateurs.

Des études spécifiques ont été réalisées au sein du laboratoire dans le but d'analyser les mécanismes de complexation de l'uranium et du neptunium avec la transferrine et ensuite d'analyser les mécanismes d'échange entre les ligands naturels et artificiels. 


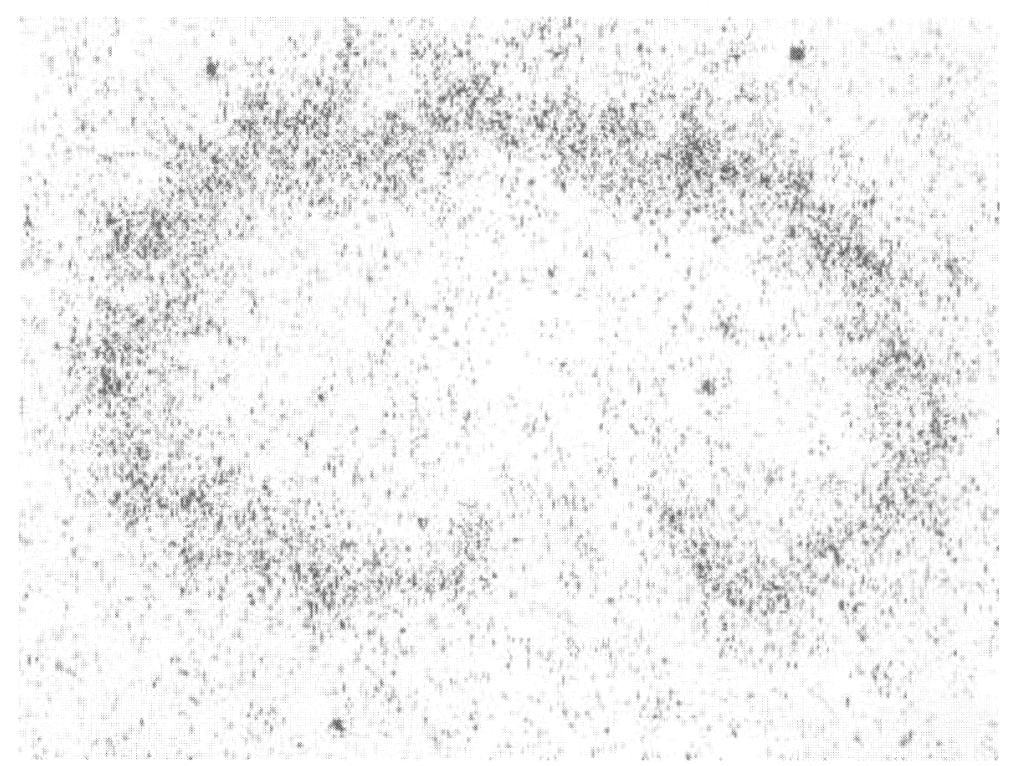

Figure 2-Autoradiographie d'une coupe longitudinale de rein de rat contaminé à l'uranium-233 (échelle : $\times 8$ ). Rats contaminés par injection intra-musculaire de $10 \mathrm{kBq}$ de ${ }^{233} \mathrm{U}$ sous forme nitrate. Euthanasie et prélèvement des reins à $\mathrm{T}+48$ heures. Activité totale des reins : $250 \mathrm{~Bq}$. Coupe de $150 \mu \mathrm{m}$ d'épaisseur, réalisée sur microtome. Image obtenue après exposition de 2 jours dans un " Cyclone » (storage phosphore system, Packard, France). L'analyse de l'image montre que 80 à $90 \%$ de l'uranium est déposé dans la zone corticale du rein.

Autoradiography of longitudinal section of rat kidney after contamination with uranium-233. (scale: $\times 8$ ). Rats were intramuscularly injected with $10 \mathrm{kBq}$ of ${ }^{233} \mathrm{U}$ as a nitrate form. Euthanasia and kidney sampling at $T+48 \mathrm{~h}$. Total renal activity: $250 \mathrm{~Bq}$. Section of 150 um using microtome. Picture obtained after a 2 days exposure in a storage phosphor system (Cyclone) Packard, France. Analysis shows that about $80 \%$ of uranium is located in the cortical part of the kidneys.

Les études effectuées dans ce domaine ont été réalisées via une modélisation informatique mais aussi via une approche expérimentale.

Les résultats obtenus dans le cas d'une modélisation simplifiée (sans tenir compte de la présence de protéines) de la spéciation de U(VI), $\mathrm{Np}(\mathrm{IV})$ et $\mathrm{Np}(\mathrm{V})$ dans le sérum sanguin montrent qu'à $\mathrm{pH} 7,4, \mathrm{U}(\mathrm{VI})$ serait sous les formes $\mathrm{UO}_{2}\left(\mathrm{CO}_{3}\right)_{3}{ }^{4-}$ et $\mathrm{UO}_{2}\left(\mathrm{CO}_{3}\right)_{2}{ }^{2-}$ (Scapolan, 1998), tandis que $\mathrm{Np}(\mathrm{V})$ et $\mathrm{Np}(\mathrm{IV})$ seraient respectivement sous forme $\mathrm{NpO}_{2}{ }^{+}$et $\mathrm{Np}\left(\mathrm{HPO}_{4}\right)_{4}{ }^{4-}$ (Racine, 2001).

L'approche expérimentale montre que U(VI) et $\mathrm{Np}(\mathrm{IV})$ sont rapidement complexés par la transferrine avec une très grande affinité $(\log \mathrm{K} \approx 16$ pour le 
complexe $2: 1 \mathrm{U}$-Tf et $\log \mathrm{K} \approx 20$ pour le complexe $1: 1 \mathrm{~Np}$-Tf). De plus, des études in vivo ont montré qu'environ $80 \%$ de $\mathrm{U}(\mathrm{VI})$ et $\mathrm{Np}(\mathrm{V})$ injectés dans le sang de rongeurs sont éliminés en moins de $3 \mathrm{~min}$, tandis que l'élimination du Np(IV) est beaucoup plus lente (environ $80 \%$ en 1 heure ; Durbin et al., 1997b).

Une compilation de ces résultats indique qu'un chélatant efficace pour U ou Np devra avoir une affinité pour les radionucléides supérieure à celles indiquées précédemment et n'aura d'action significative que sur la fraction recyclée du radionucléide.

Des études complémentaires visant à expliquer les échanges entre les ligands naturels et artificiels ont été réalisées in vitro avec les complexes Pu-Tf et $\mathrm{Np}$-Tf, en présence de différents ligands tels que le DTPA, LICAM ${ }^{\circledR}$ et Li-HOPO. Le DTPA s'est avéré moins efficace que le LICAM $^{\oplus}$ pour dissocier le complexe PuTf (Métivier et al., 1983), tandis que le DTPA et le Li-HOPO se sont avérés inefficaces pour dissocier le complexe $\mathrm{Np}$-Tf (Paquet et al., 1997, 1998a). Ces études, réalisées au niveau moléculaire, expliquent pourquoi Np est résistant aux thérapies usuelles et ont guidé les investigations vers de nouvelles voies.

\section{Conclusions}

Les études de spéciation sont nécessaires à l'amélioration des connaissances dans de nombreux domaines de la dosimétrie interne. Ces études et leurs résultats peuvent aider à mieux décrire, comprendre et donc prévoir les biocinétiques et la toxicité des radionucléides. La plus grande difficulté dans les études de spéciation réside dans le choix de la technique la plus fiable en fonction de la précision demandée et du degré d'incertitude toléré. Étant donné qu'il n'existe aucune technique universelle conçue pour fournir des données fiables en toutes circonstances, différentes techniques complémentaires devront être combinées afin de déterminer la spéciation des éléments avec le moins d'incertitude possible. Cependant, du fait de la complexité de la plupart des techniques et du nombre très important des formes chimiques potentielles des radionucléides, les études de spéciation ne devront pas être entreprises de façon générique mais plutôt sur des tissus spécifiques, comme ceux impliqués dans l'entrée, le stockage ou l'excrétion des radionucléides et sur des molécules spécifiques, déjà décrites comme complexant les métaux.

En dépit de ces restrictions, une telle tâche reste une ambition importante qui nécessite une approche pluridisciplinaire regroupant des compétences dans de nombreux domaines tels que la chimie, la biochimie, la biologie, la médecine, la toxicologie et la pharmacologie. 


\section{RÉFÉRENCES}

Bethke C.M. (1996) Geochemical Reaction Modeling: Concepts and Applications, 397 pages. Oxford University Press, New-York.

Boocock G., Danpure C.J., Popplewell D.S., Taylor D.M. (1970) The subcellular distribution of plutonium in rat liver, Radiat. Res. 42, 381-396.

Bruenger F.W., Stover B.J., Stevens W. (1971) ${ }^{239}$ Pu(IV): Its subcellular distribution and association with ferritin in the canine liver, Health Phys. 21, 679-687.

Bruenger F.W., Atherton D.R., Stevens W. (1972) Intracellular distribution of ${ }^{249} \mathrm{Cf}$ in canine liver, Health Phys. 22, 685-689.

Cornelis R., De Kimpe J., Zhang X. (1998) Trace elements in clinical samples revisited-speciation is knocking at the door. Sample preparation, separation of the species and measurements methods, Spectrochitn. Acta 53, 187-196.

Durbin P.W., Kullgren B., Schmidt C.T. (1997a) Circulatory kinetics of intravenously injected ${ }^{238} \mathrm{Pu}(\mathrm{IV})$ citrate and ${ }^{14} \mathrm{C}$-CaNa3-DTPA in mice comparison with rat, dog, and reference man, Health Phys. 72, 222-235.

Durbin P.W., Kul]gren B., Xu J., Raymond K.N. (1997b) New agents for in vivo chelation of uranium(VI): efficacy and toxicity in mice of multidentate catecholate and hydroxypyridonate ligands, Health Phys. 72, 865-879.

Eisele G.R., Erickson B.H. (1985) Gonadal retention and localization of ${ }^{241} \mathrm{Am}$ in orally exposed swine, Health Phys. 49, 1276-1279.

Flanagan P.R., McLellan J.S., Haist J., Cherian G., Chamberlain M.J., Valberg L.S. (1978) Increased dietary cadmium absorption in mice and human subjects with iron deficiency, Gastroenterology 74, $841-846$.

Fuger J., Khodakovsky I.L., Sergeyeva E.I., Medvedev V.A. (1992) The chemical thermodynamic of actinide elements, Part 12, The actinide aqueous inorganic complexes, Vienna, IAEA.

Goyer R.A. (1983) Metal-protein complexes in detoxification processes, in: Chemical Toxicology and clinical chemistry of metals (Brown S.S., Savory J., Eds.) pp. 199-209. Academic Press, London.

Grenthe I., Fuger J., Lemire R., Muller R.J., Nguyen-Trung C., Wanner H. (1992) Chemical thermodynamics of uranium, in: OECD Nuclear Energy Agency. North Holland, Amsterdam London - New York - Tokyo.

Guilmette R.A., Edison A.F. (1992) Using animal dosimetry models to interpret human bioassay data for actinide exposures, J. Radioanalyt. Nucl. Chem. 156, 425-449.

Jones P.W., Taylor D.M., Webb L., Williams D.R. (2002) Computer modelling of the chemical speciation of caesium, uranium (VI) and neptunium (V) in human duodenal fluids under fasting conditions, Appl. Radiat. Isot. 57, 159-165.

Jonnalagadda S.B., Rao P.V. (1993) Toxicity, bioavailability and metal speciation, Comp. Biochem. Physiol. C 106, 585-595.

Lana C. (2000) Modélisation de la spéciation de l'uranium dans le tractus gastrointestinal, pp. 1-67, rapport ENSCP, École nationale supérieure de chimie de Paris, France.

Lehmann M., Culig H., Taylor D.M. (1983) Identification of transferrin as the principal plutoniumbinding protein in the blood serum and liver cytosol of rats: immunological and chromatographic studies, Int. J. Radiat. Biol. Relat. Stud. Phys. Chem. Med. 44, 65-74.

Martell A.E., Calvin M. (1959) Chemistry of the Metal Chelate Compounds (Latimer W., Ed.). M. Pentice-Hall Chemistry series. 


\section{SPÉCIATION ET DOSIMÉTRIE INTERNE}

Masse R., Métivier H., Guillaumont R. (1973) Physico-chimie et cristallographie des apatites d'intérêt Biologique, Fixation osseuse des terres rares et des éléments transuraniens, 10-15 septembre 1973, Paris, $N^{\circ} 230$, Centre National de la Recherche Scientifique, pp. 441-450.

May P.M., Murray K. (1991) JESS, A joint expert speciation system - I. Raison d'être, Talanfa 38, 1409-1417.

McInroy J.F., Boyd H.A., Eutsler B.C., Romero D. (1985) The U.S. Transuranium Registry report of the ${ }^{24 i}$ Am content of a whole body. Part IV: Preparation and analysis of the tissues and bones, Health Phys. 49, 587-621.

Métivier H., Masse R., Lafuma J. (1983) Metabolism of plutonium introduced as tri-n-butylphosphate complex in the rat and removal attempts by DTPA, Health Phys. 44, 623-634.

Mirto H., Barrouillet M.P., Henge-Napoli M.H., Ansoborlo E., Fournier M., Cambar J. (1999) Influence of uranium (VI) speciation for the evaluation of in vitro uranium cytotoxicity on LLCPKI cells, Hum. Exp. Toxicol. 18, 180-187.

Paquet F., Verry M., Grillon G., Landesman C., Masse R., Taylor D.M. (1995) Subcellular and intranuclear localization of neptunium-237 (V) in rat liver, Radiat. Res. 143, 214-218.

Paquet F., Ramounet B., Métivier H., Taylor D.M. (1996) The effect of the mass and initial chemical form of neptunium on its molecular associations in blood and liver, Radiat. Res. 146, 306-312.

Paquet F., Métivier H., Poncy J.L., Burgada R., Bailly T. (1997) Evaluation of the efficiency of DTPA and other new chelating agents for removing neptunium from target organs, Int. J. Radiat. Biol. 71, 613-62I.

Paquet F., Ramounet B., Hengé-Napoli M.H., Métivier H., Gourmelon P. (1998a) In vitro experiments to determine the mechanisms by which chelators remove the actinides neptunium and uranium from the body, Radiat. Prot. Dos. 79, 467-470.

Paquet F., Ramounet B., Métivier H., Taylor D.M. (1998b) The bioinorganic chemistry of Np, Pu and Am in mammalian liver, J. Alloys Comp. 271-273, 85-88.

Parkhurst D.L., Thorstenson D.C., Plummer L.N. (1980) PHREEQE - a computer program for geochemical calculations., USGS/WRD/WRI-81-018 report, pp. 1-218.

Popplewell D.S., Stradling G.N., Ham G.J. (1975) The chemical form of plutonium in urine, Radiat. Res. 62, 513-519.

Powell K. (1999) The stability constant database. Academic software and IUPAC (1992-1999) (software).

Racine R. (2001) Etude des mécanismes de complexation du neptunium en milieu biologique en vue de la mise au point d'une thérapeutique applicable en cas d'incorporation accidentelle, Thèse de doctorat de l'université Paris XI. 189 pp.

Sabbioni E., Fischbach M., Pozzi G., Pietra R., Gallorini M., Piette J.L. (1991) Cellular retention, toxicity and carcinogenic potential of seafood arsenic. I. Lack of cytotoxicity and transforming activity of arsenobetaine in the BALB/3T3 cell line, Carcinogenesis 12, 1287-1291.

Sasser L.B., Mahlum D.D., Rommereim R.L. (1986) Influence of pregnancy and lactation on maternal deposition and perinatal uptake of ${ }^{241} \mathrm{Am}$ in the rat, Health Phys. 50, 595-604.

Savory J., Bertholf R.L., Wills M.R. (1983) Chemical toxicology and clinical chemistry of metals, Speciation and fractionation of toxic metals in biological material (Brown S.S., Savory J., Eds.) pp. 183-198. Academic Press, London.

Scapolan S. (1998) Mise au point et évaluation de techniques de spéciation pour l'étude des espèces biologiques circulantes de l'uranium (VI), Thèse de doctorat de l'université Paris X1.

Scapolan S., Ansoborlo E., Moulin C., Madic C. (1997) Uranium speciation in biological medium by means of capillary electrophoresis and time-resolved laser-induced fluorescence, $J$. Radioanal. Nucl. Chem. 226, 145-148. 
Schuler F., Taylor D.M. (1987) The subcellular distribution of ${ }^{238} \mathrm{Pu}$ and ${ }^{239} \mathrm{Pu}$ in primary cultures of rat hepatocytes, Radiat. Res. 110, 362-371.

Schuppler U., Planas-Bohne F., Taylor D.M. (1988) Biochemical binding and distribution of protactinium-233 in the rat, Int. J. Radiat. Biol. Relat. Stud. Phys. Chem. Med. 53, 457-466.

Seidel A., Kruger E., Wiener M., Hotz G., Balani M., Thies W.G. (1985) Association of ${ }^{239} \mathrm{Pu}$ with lysosomes in rat, Syrian hamster and Chinese hamster liver as studied by carrier-free electrophoresis and electron microscopic autoradiography with ${ }^{241} \mathrm{Pu}$, Radiat. Res. 104, 191199.

Simkiss K., Taylor M.G. (2001) Trace element speciation at cell membranes: aqueous, solid and lipid phase effects, I. Environ. Monit. 3, 15-21.

Stradling G.N., Popplewell D.S., Ham G.J. (1976) The chemical form of americium and curium in urine, Health Phys. 31, 517-519.

Sutterlin U., Thies W.G., Haffner H., Seidel A. (1984) Comparatives studies on the lysosomal association of monomeric ${ }^{239} \mathrm{Pu}$ and ${ }^{241} \mathrm{Am}$ in rat and Chinese hamster liver: analysis with sucrose, metrizamide and percoll density gradient of subcellular binding as dependant on time, Radiat. Res. 98, 293-306.

Taylor D.M. (1989) The biodistribution and toxicity of plutonium, americium and neptunium, Sci. Total Environ. 83, 217-225.

Taylor D.M., Seidel A., Planas-Bohne F., Schuppler U., Neu-Muller M., Wirth R. (1987) Biochemical studies of the interactions of plutonium, neptunium and protactinium with blood and liver cell proteins, Inorg. Chim. Acta 140, 361-363.

Taylor G.N., Lloyd R.D., Mays C.W., Angus W., Miller S.C., Shabestari L.R., Hahn F.F. (1991) Plutonium- or americium-induced liver tumors and lesions in beagles, Health Phys. 61, 337347.

Templeton D.M., Ariese F., Comelis R., Danielson L.G., Muntau H., Van Leeuwen H.P., Lobinski R. (2000) Guidelines for terms related to chemical speciation and fractionation of elements. Definitions, structural aspects and methodological approaches (IUPAC recommendations 2000), Pure Appl. Chem. 72, 1453-1470.

Toohey R.E., Essling M.A. (1980) Measurements of ${ }^{241} \mathrm{Am}$ in vivo at long times after inhalation, Health Phys. 38, 139-145.

Tseveleva I.A. (1960) The plutonium content in the protein fraction of rat tubular bones, Biokhimiya 25, 636-639.

Turner G.A., Taylor D.M. (1968) The binding of plutonium to serum proteins in vitro, Radiat. Res. 36, 22-30.

Van den Heuvel R.L., Gerber G.B., Leppens H., Van der Plaetse F., Schoeters G.E. (1995) Long-term effects on tumour incidence and survival from ${ }^{241} \mathrm{Am}$ exposure of the $\mathrm{BALB} / \mathrm{c}$ mouse in utero and during adulthood, Int. J. Radiat. Biol. 68, 679-686.

Van der Lee J. (1998) Thermodynamic and Mathematical concepts of CHESS, Technical report LHM/RD/98/39, pp. 1-78.

Westall J.C., Zachary J.L., Morel F.M. (1986) MINEQL. A computer program for the calculation of the chemical equilibrium composition of aqueous systems, Department of Chemistry, Oregon State University, Corvalis, OR Report 86-01,

Whanger P.D. (1992) Selenium in the treatment of heavy metal poisoning and chemical carcinogenesis, J. Trace Elem. Electrolytes Health Dis. 6, 209-221.

Zalikin G.A., Nisimov P.G. (1990) The effect of age on the distribution of ${ }^{241}$ Am in the rat body, Radiobiologiya. 30, 422-424. 